\title{
Resilienz im Vorschulalter: Wie stark kann die familiäre Leseumwelt biologische und soziokulturelle Entwicklungsrisiken kompensieren?
}

\section{Resilience in preschool children: To what degree can a stimulating home literacy environment compensate biological and socio-cultural adversity?}

\section{Zusammenfassung:}

Erfolgreiche kindliche Entwicklung kann durch biologische und soziokulturelle Risiken behindert werden. Zugleich zeigen zahlreiche Studien, dass die familiäre Leseumwelt einen protektiven Faktor für die Entwicklung im Vorschulalter darstellen kann. Wir verglichen Daten aus zwei Längsschnittstudien um zu untersuchen, wie stark die familiäre Leseumwelt biologische (Frühgeburtlichkeit) und soziokulturelle Risiken (niedriger sozioökonomischer Status und Migrationshintergrund) in der kognitiven und sprachlichen Entwicklung von Vorschulkindern kompensieren kann. Unsere Ergebnisse zeigten, dass die häusliche Leseumwelt positive Effekte auf die Entwicklung sowohl türkischstämmiger als auch sehr früh und/oder sehr leicht geborener Kinder ausübt. Dies bedeutet, dass Eltern von Vorschulkindern mit soziokulturellem oder biologischem Risiko Resilienz fördern können, indem sie ihnen eine reichhaltige Leseumwelt zur Verfügung stellen. Wir benötigen mehr Informationen über die spezifischen Bedürfnisse von Kindern, die mit unterschiedlichen Entwicklungsrisiken aufwachsen, um diesen eine bessere Förderung zu ermöglichen.

Schlagwörter: biologische und soziokulturelle Risiken, Resilienz, kognitive und sprachliche Entwicklung, familiäre Leseumwelt

\begin{abstract}
:
Positive child development may be impeded by biological and socio-cultural adversity. However, a cognitively stimulating environment may compensate these risks. Studies have confirmed positive effects of preschool children's home literacy environments for their school readiness. We compared data of two longitudinal studies to investigate to what degree a stimulating home literacy environment can compensate biological (premature birth) and socio-cultural adversity (low SES and migration background). Results showed that a more stimulating home literacy environment predicted better cognitive and language development of both Turkish immigrant and very preterm /very low birth weight preschool children. Thus, parents of biological or socio-cultural at-risk children can promote resilience by providing a stimulating home literacy environment. We need more data on the specific developmental needs of different groups of at-risk children to facilitate their academic achievement.
\end{abstract}

Key words: biological and socio-cultural adversity, resilience, cognitive and language development, home literacy environment 


\section{Kindliche Entwicklung und Resilienz}

Frühkindliche Entwicklung ist durch spezifische normative Entwicklungsaufgaben definiert. Von Vorschulkindern wird zum Beispiel erwartet, dass sie bestimmte Basisfähigkeiten erwerben, die sie auf die Schule vorbereiten. Dies kann durch biologische (z.B. eine zu frühe Geburt) und soziokulturelle Risiken (z.B. ein niedriger sozioökonomischer Status (SES) und Migrationshintergund) behindert werden. Einige Kinder entwickeln sich jedoch auch unter adversiven Bedingungen positiv (Cicchetti 2010; Masten 2007). Kindliche Resilienz kann durch protektive Faktoren in der familiären Umwelt gefördert werden, zum Beispiel stellen regelmäßiges Vorlesen und gemeinsame Spiele wichtige Ressourcen dar (Jaekel et al. 2011). Darüber hinaus beeinflussen Eltern den späteren Schulerfolg, indem sie Basisfertigkeiten (z.B. zuzuhören, wenn andere sprechen; bis zehn zählen können) vermitteln, die die Partizipation am Schulunterricht ermöglichen (Arnold 2003). Kinder, denen regelmäßig vorgelesen wird, zeigen ein größeres Interesse an Büchern und zahlreiche Studien untermauern die Bedeutung der häuslichen Leseumwelt für die sprachliche und kognitive Entwicklung (Bus et al. 1995; Mol et al. 2008; Raikes et al. 2006; Whitehurst et al. 1994).

Unklar ist bisher, inwieweit die familiäre Leseumwelt biologische im Vergleich zu soziokulturellen Entwicklungsrisiken kompensieren kann. Wir wissen, dass frühe MutterKind-Interaktionen Resilienz im kindlichen Verhalten fördern können (DeWolff/van Ijzendoorn 1997; Laucht et al. 2002). Individuelle kindliche Charakteristika führen jedoch zu einer unterschiedlich hohen Empfänglichkeit für Umwelteinflüsse (Belsky 1997; Belsky/Pluess 2009). Um Kindern, die mit biologischen oder soziokulturellen Entwicklungsrisiken aufwachsen, eine erfolgreiche Schullaufbahn zu ermöglichen, benötigen wir mehr Informationen über das Zusammenspiel von Risiko- und Schutzfaktoren und deren Wirkung auf den kindlichen Entwicklungsstand vor dem Schuleintritt.

\section{Soziokulturelles Risiko}

Ungefähr ein Drittel aller Kinder in den alten Bundesländern haben einen Migrationshintergrund (Citlak et al. 2008; Gogolin et al. 2003). Eine Migration stellt nicht unbedingt ein Risiko für die kindliche Entwicklung dar, jedoch sind in Deutschland ethnische Herkunft und sozioökonomischer Status häufig konfundiert, so dass Kinder mit türkischem Migrationshintergrund überwiegend aus Familien mit niedrigem sozioökonomischem Status kommen (Leyendecker/Schölmerich 2005). Die Zukunftschancen dieser Kinder sind abhängig von einer erfolgreichen Vorbereitung auf ihre spätere Schulkarriere (Leyendecker 2003). In unserer Forschung zu den Entwicklungsbedingungen türkischer Migrantenkinder versuchen wir, zwischen Einflüssen von Kultur und Einflüssen von Bildung zu unterscheiden. Einerseits ist die Qualität der häuslichen Leseumgebung stark vom Bildungshintergrund der Eltern beeinflusst (Jaekel et al. 2011), andererseits ist Vorlesen eine kulturell geprägte Aktivität (Kim 2009) und nicht unbedingt Teil der tradierten Verhaltensweisen von Eltern mit Migrationshintergrund (Bus et al. 2000; Bus/Sulzby 1996). Mütter mit türkischem Migrationshintergrund lesen ihren Kindern im Vergleich zu deutschen Müttern durchschnittlich seltener vor (Driessen et al. 2009; Jaekel et al. 2011). In 
Bezug auf die kindliche Entwicklung gilt, dass Effekte des mütterlichen Migrationshintergrunds durch die Häufigkeit des Vorlesens positiv beeinflusst werden (Glick et al. 2009; Leyendecker et al. 2011). Kinder, die in Familien mit niedrigem SES und türkischem Migrationshintergrund aufwachsen, haben daher ein doppeltes Risiko, da sie zu Hause gegebenenfalls wenige Lerngelegenheiten erhalten. Langfristig kann dies zur Folge haben, dass sie schlechte Schulleistungen zeigen und hierdurch ihre Chancen auf gesellschaftliche Teilhabe gemindert werden.

\section{Biologisches Risiko}

Biologische Entwicklungsrisiken wie eine zu frühe Geburt können ebenso wie soziokulturelle Risiken zu schulischen Problemen führen, die zu Grunde liegenden Mechanismen sind aber unterschiedlicher Natur. Das menschliche Gehirn reagiert sehr sensibel auf die Konsequenzen einer zu frühen Geburt (Rees/Inder 2005). Abhängig vom Gestationsalter ist die Organ- und Hirnentwicklung Frühgeborener unreif und eine Reorganisation kortikaler Strukturen noch im Jugendalter nachweisbar (Nosarti et al. 2010). Frühgeborene haben ein höheres Risiko für Probleme in kognitiven Leistungen, im Sozialverhalten und in ihrer Aufmerksamkeitsregulation als reif geborene Kinder (Aarnoudse-Moens et al. 2009; Hall et al. 2012, in Druck; Johnson 2007; Wolke 1998). Diese Fähigkeiten stellen entscheidende Faktoren für kindliche Lernfortschritte und späteren Schulerfolg dar (Jaekel et al. 2012, in Druck; Johnson et al. 2009; Mulder et al. 2010; Schneider et al. 2004; Taylor et al. 2011). Neuere Studien zeigen ein graduelles Risiko in Abhängigkeit vom Gestationsalter: zum Beispiel entwickelt nur ein kleiner Teil der moderat Frühgeborenen (Geburt in der 32.-36. Schwangerschaftswoche) schulische Probleme, während jede verlorene Woche das Entwicklungsrisiko der sehr $(<32$ Wochen) oder extrem früh $(<26$ Wochen) geborenen Kinder bedeutend erhöht (Quigley et al. 2012; Saigal et al. 2003). Viele Eltern und Lehrer erwarten, dass ehemalige Frühgeborene den Entwicklungsrückstand zu Gleichaltrigen bis zum Schuleintritt aufholen. Während dies auf die Mehrheit zutrifft, haben Frühgeborene mit hohem Entwicklungsrisiko oft noch langfristig Probleme, da sie eine eingeschränkte Plastizität besitzen und weniger von Lerngelegenheiten profitieren können. Frühgeborene, die in Familien mit niedrigem SES aufwachsen, haben ein doppeltes Risiko (Wolke et al. 2001), denn sie benötigen zusätzliche Unterstützung, um ihr Entwicklungspotential zu entfalten, erhalten aber zu Hause häufig weniger reichhaltige Lerngelegenheiten. Um erfolgreiche Interventionen zu entwickeln, müssen wir die besonderen kindlichen Bedürfnisse kennen und berücksichtigen. Zum Beispiel können familiäre Faktoren wie die Qualität der Eltern-Kind Interaktionen (Forcada-Guex et al. 2006) und eine kognitiv anregende Umgebung (Bradley et al. 1995; Weisglas-Kuperus et al. 1993) die kindliche Entwicklungsprognose positiv beeinflussen. Diese Studien lassen vermuten, dass auch Eltern von Kindern mit hohem biologischem Risiko aktiv einen erfolgreichen Schuleintritt fördern können, indem sie reichhaltige Lerngelegenheiten zur Verfügung stellen. 


\section{Fragestellung}

Biologische und soziokulturelle Risiken behindern die kindliche Entwicklung. Wir wissen jedoch nicht sicher, wie stark protektive Faktoren wie die familiäre Leseumwelt in Populationen mit unterschiedlichen Entwicklungsrisiken wirken, da es eine Rolle spielt, ob die jeweiligen Entwicklungsrisiken in der Umwelt oder im Kind selber begründet sind. In den meisten Studien werden längsschnittliche Fragen an einer einzigen Stichprobe getestet; um Effekte biologischer und soziokultureller Risiken getrennt zu untersuchen, können stichprobenübergreifende Analysen jedoch sinnvoller sein (Feldman 2007), da der Einfluss auf die individuelle kindliche Plastizität je nach Schwere und Art des Risikos unterschiedlich ist. Wir vergleichen Daten aus zwei Längsschnittstudien, um zu untersuchen, wie stark die familiäre Leseumwelt biologische und soziokulturelle Risiken in der kognitiven und sprachlichen Entwicklung von Vorschulkindern kompensieren kann.

\section{Methoden}

\section{Stichprobe 1 (soziokulturelles Risiko):}

Für die vorliegende Studie wurden türkischstämmige Familien mit Kindern im Alter von vier Jahren aus Kindergärten im Ruhrgebiet geworben. Die Rekrutierung erfolgte über eine Kontaktaufnahme zu den jeweiligen Kindergartenleitungen, die die Mütter über die Studie informierten. Die Teilnahme war freiwillig und wurde mit einer Aufwandsentschädigung vergolten. Die Datenerhebungen fanden entweder bei den Familien zu Hause oder in den Kindergärten durch zweisprachige Interviewerinnen türkischer Herkunft statt. Die türkischstämmigen Mütter $(n=75)$ waren zum ersten Erhebungszeitpunkt durchschnittlich 30 Jahre alt. Die überwiegende Mehrheit der Mütter war verheiratet und lebte mit ihrem Ehepartner zusammen, drei Mütter waren alleinerziehend. Unter den türkischstämmigen Kindern waren 35 Mädchen und 40 Jungen. Da es große Unterschiede im Bildungsstand der türkischstämmigen Mütter gab, wurde die Bildung für die weiteren Analysen in zwei Untergruppen differenziert: 20 Mütter hatten keine oder nur eine geringe Schulbildung zwischen null und neun Jahren, während 55 Mütter mindestens zehn Jahre eine Schule besucht hatten.

Instrumente Stichprobe 1

Zum ersten Erhebungszeitpunkt (im Alter von 4 Jahren) beantworteten die Mütter Fragen zu ihren demographischen Daten sowie zur häuslichen Leseumwelt (für eine detaillierte Beschreibung siehe Jäkel et al., 2011). Es wurde erhoben, wie häufig die Eltern dem Kind vorlasen, wie häufig sie selber lasen, und wie viele Kinderbücher das Kind besaß. Aus diesen Informationen wurde der Home Literacy Environment (HLE)-Summenscore gebildet (Jaekel, et al. 2011). Mit den Kindern wurde der SETK 3-5 (Grimm et al. 2001) durchgeführt, der rezeptive und produktive Sprachverarbeitungsfähigkeiten sowie auditive Gedächtnisleistungen erfasst und für den Einsatz bei Kindern mit Deutsch als Zweitsprache geeignet ist (Caspar/Leyendecker 2011). Aus den drei Subtests Verstehen von 
Sätzen, Morphologische Regelbildung und Satzgedächtnis wurde für die weiteren Analysen ein SETK-Gesamtscore gebildet.

Im Alter von sechs Jahren wurden die kognitiven Fähigkeiten der Kinder mit dem ET 6-6 (Petermann et al. 2006) erhoben. Der ET 6-6 erfasst den altersspezifischen Entwicklungsstand und ist mit Einschränkungen auch für den Einsatz in türkischstämmigen Stichproben geeignet (Jaekel, et al. 2011; Schölmerich et al. 2008).

Stichprobe 2 (biologisches Risiko):

Die hier untersuchten Daten wurden im Rahmen der Bayrischen Entwicklungsstudie $(B E S t){ }^{1}$ erhoben (Riegel et al. 1995; Schneider, et al. 2004; Wolke et al. 2009). Die Eltern wurden in den ersten Tagen nach der Geburt eines sehr früh $(<32$. Schwangerschaftswoche) und/oder sehr leicht (Geburtsgewicht $<1500 \mathrm{~g}$ ) geborenen Kindes für die Teilnahme geworben und im Krankenhaus interviewt. Da der sozioökonomische Status elterliches Verhalten und kindliche Entwicklung beeinflusst und das Bildungsniveau in Stichprobe 1 teilweise sehr niedrig war, wählten wir für die aktuellen Analysen aus Stichprobe 2 diejenigen Mütter aus, deren Bildungsstand dem der türkischstämmigen Mütter aus Stichprobe 1 entsprach. Demnach wurde die Bildung in zwei Untergruppen differenziert: 31 Mütter besaßen keinen Schulabschluss oder einen Hauptschulabschluss, während 99 Mütter die Mittlere Reife oder das (Fach-)Abitur erworben hatten. Die Mütter $(n=130)$ waren zum Zeitpunkt der Geburt des Kindes durchschnittlich 28 Jahre alt. Die Mehrheit der Mütter lebte mit einem (Ehe-)Partner, zehn Mütter waren alleinerziehend. Unter den sehr früh geborenen Kindern waren 66 Mädchen und 64 Jungen.

Instrumente Stichprobe 2

Gestationsalter und Geburtsgewicht der Kinder waren in den Krankenhausakten dokumentiert. In den ersten zehn Tagen nach der Geburt beantworteten die Mütter Fragen zu ihren demografischen Daten.

Im korrigierten Alter von sechs Jahren wurden die kindlichen kognitiven Fähigkeiten mit der deutschen Version der Kaufman Assessment Battery for Children (K-ABC) (Kaufman/Kaufman 1983; Melchers/Preuss 1991) erhoben. Für die weiteren Analysen wurde die Skala Intellektuelle Fähigkeiten (SIF) verwendet. Zum gleichen Zeitpunkt wurde mit den Kindern der Heidelberger Sprachentwicklungstest (HSET) (Grimm/Schöler 1991) durchgeführt. Aus den Untertests Verstehen grammatischer Strukturen, Singular-Plural-Bildung, Korrektur semantisch inkonsistenter Sätze, sowie Satzbildung wurde ein HSET-Gesamtscore gebildet (Wolke/Meyer 1999). Die Mütter beantworteten Fragen zur häuslichen Leseumwelt (wie häufig lesen die Eltern dem Kind vor, wie häufig lesen sie selber, wie viele Kinderbücher besitzt das Kind), aus denen ein Summenscore gebildet wurde.

1 Die Bayerische Entwicklungsstudie wurde gefördert durch die Programmförderung des Bundesministeriums für Bildung und Forschung: PKE24, JUG14, 01EP9504 und 01ER0801. Der Artikel liegt in der Verantwortung der Autoren und stellt nicht die offizielle Meinung des Förderers dar. 


\section{Ergebnisse}

Wir sind im Rahmen dieser Studie an den Mechanismen interessiert, die den Einfluss der familiären Leseumwelt auf die kindliche Entwicklung bei Vorhandensein von biologischen oder soziokulturellen Risiken kennzeichnen. Deshalb haben wir parallel für beide Stichproben und getrennt nach Bildungsgruppen Regressionsanalysen durchgeführt. Diese stellen die zentralen Ergebnisse dar. Wir haben auf einen Vergleich der Mittelwerte in den Substichproben verzichtet, da Gruppenunterschiede in Leistungsniveau und Qualität der Leseumwelt nicht im Fokus der Studie stehen.

Tabelle 1 zeigt die Ergebnisse der Regressionsanalysen für den Einfluss der häuslichen Leseumwelt auf die kognitive und sprachliche Entwicklung türkischstämmiger Vorschulkinder, getrennt nach niedriger und mittlerer mütterlicher Bildung und kontrolliert für Geschlecht, Familienstand und Alter der Mutter. Die häusliche Leseumwelt übte einen signifikanten positiven Effekt auf die kognitive und sprachliche Entwicklung der türkischstämmigen Kinder aus, deren Mütter mindestens zehn Jahre eine Schule besucht hatten, hatte aber keine signifikanten Effekte auf die Entwicklung der türkischstämmigen Kinder, deren Mütter weniger als zehn Jahre eine Schule besucht hatten.

Tabelle 1: Regressionsanalysen für den Einfluss der häuslichen Leseumwelt (Schritt 2) auf die kognitive und sprachliche Entwicklung türkischstämmiger Vorschulkinder, kontrolliert für Geschlecht, Familienstand und Alter der Mutter (Schritt 1)

\begin{tabular}{|c|c|c|c|c|c|c|c|c|}
\hline & \multicolumn{4}{|c|}{$\begin{array}{l}\text { Türkischstämmige Kinder, } \\
\text { niedrige mütterliche Bildung } \\
(n=20)\end{array}$} & \multicolumn{4}{|c|}{$\begin{array}{l}\text { Türkischstämmige Kinder, } \\
\text { mittlere mütterliche Bildung } \\
\qquad(n=55)\end{array}$} \\
\hline & $R^{2}$ & $\Delta R^{2}$ & $\Delta F$ & $\beta$ & $R^{2}$ & $\Delta R^{2}$ & $\Delta F$ & $B$ \\
\hline \multicolumn{9}{|c|}{ AV: Kognitive Entwicklung 6 J. (ET 6-6) } \\
\hline (1) & .46 & .46 & $7.11^{* *}$ & & .07 & .07 & 1.28 & \\
\hline Geschlecht & & & & .31 & & & & $-.22^{\circ}$ \\
\hline Familienstand & & & & - & & & & .01 \\
\hline Alter der Mutter & & & & $.53^{*}$ & & & & .05 \\
\hline (2) Häusliche Leseumwelt $4 \mathrm{~J}$. & .46 & .00 & 0.13 & .07 & .17 & .10 & $6.02^{*}$ & $.33^{*}$ \\
\hline \multicolumn{9}{|c|}{ AV: Sprachliche Entwicklung 4 J. (SETK) } \\
\hline (1) & .13 & .13 & 1.01 & & .05 & .05 & .79 & \\
\hline Geschlecht & & & & .23 & & & & -.31 \\
\hline Familienstand & & & & - & & & & .04 \\
\hline Alter der Mutter & & & & .27 & & & & .01 \\
\hline (2) Häusliche Leseumwelt $4 \mathrm{~J}$. & .29 & .17 & 3.09 & -.43 & .19 & .14 & $8.48^{\star *}$ & $.35^{\star *}$ \\
\hline
\end{tabular}

${ }^{\circ}=p<.10 ; *=p<.05 ; * *=p<.01$; Geschlecht: $1=$ männlich, $2=$ weiblich; Familienstand: $1=$ zusammenlebend, 2 = alleinerziehend (in der Gruppe mit niedriger mütterlicher Bildung konstant, da alle zusammenlebend)

Tabelle 2 zeigt die Ergebnisse der parallel durchgeführten Regressionsanalysen für den Einfluss der häuslichen Leseumwelt auf die kognitive und sprachliche Entwicklung sehr früh und/oder sehr leicht geborener Vorschulkinder, getrennt nach niedriger und mittlerer mütterlicher Bildung und kontrolliert für Geschlecht, Familienstand und Alter der Mutter. 
Ebenso wie bei den türkischstämmigen Kindern übte die häusliche Leseumwelt einen signifikanten positiven Effekt auf die kognitive und sprachliche Entwicklung derjenigen sehr früh und/oder sehr leicht geborenen Kinder aus, deren Mütter eine mittlere Bildung erworben hatten, hatte aber keine signifikanten Effekte auf die Entwicklung der Kinder, deren Mütter weniger als zehn Jahre eine Schule besucht hatten. Darüber hinaus übte das Geschlecht einen signifikanten Einfluss auf die Sprachfähigkeiten aus: Mädchen zeigten bessere Leistungen als Jungen.

Tabelle 2: Regressionsanalysen für den Einfluss der häuslichen Leseumwelt (Schritt 2) auf die kognitive und sprachliche Entwicklung sehr früh und/oder sehr leicht geborener Vorschulkinder, kontrolliert für Geschlecht, Familienstand und Alter der Mutter (Schritt 1)

\begin{tabular}{|c|c|c|c|c|c|c|c|c|}
\hline & \multicolumn{4}{|c|}{$\begin{array}{l}\text { Sehr früh/leicht geborene Kinder, } \\
\text { niedrige mütterliche Bildung }(n=31\end{array}$} & \multicolumn{4}{|c|}{$\begin{array}{l}\text { Sehr früh/leicht geborene Kinder, } \\
\text { mittlere mütterliche Bildung }(n=99)\end{array}$} \\
\hline & $R^{2}$ & $\Delta R^{2}$ & $\Delta F$ & $\beta$ & $R^{2}$ & $\Delta R^{2}$ & $\Delta F$ & $\beta$ \\
\hline \multicolumn{9}{|c|}{ AV: Kognitive Entwicklung 6 J. (K-ABC) } \\
\hline$(1)$ & .07 & .07 & .66 & & .02 & .02 & .56 & \\
\hline Geschlecht & & & & .15 & & & & .03 \\
\hline Familienstand & & & & -.27 & & & & .12 \\
\hline Alter der Mutter & & & & -.09 & & & & .06 \\
\hline (2) Häusliche Leseumwelt $6 \mathrm{~J}$. & .09 & .02 & .52 & .15 & .06 & .05 & $4.64^{*}$ & $.22^{*}$ \\
\hline \multicolumn{9}{|c|}{ AV: Sprachliche Entwicklung 6 J. (HSET) } \\
\hline (1) & .06 & .06 & .59 & & .07 & .07 & $2.37^{\circ}$ & \\
\hline Geschlecht & & & & -.02 & & & & $.22^{*}$ \\
\hline Familienstand & & & & .09 & & & & $.19^{\circ}$ \\
\hline Alter der Mutter & & & & .07 & & & & .05 \\
\hline (2) Häusliche Leseumwelt $6 \mathrm{~J}$. & .18 & .12 & $3.62^{\circ}$ & $.37^{\circ}$ & .13 & .06 & $6.98^{\star *}$ & $.26^{* *}$ \\
\hline
\end{tabular}

${ }^{\circ}=p<.10 ; *=p<.05 ; * *=p<.01$; Geschlecht: $1=$ männlich, $2=$ weiblich; Familienstand: $1=$ zusammen lebend, 2 = allein erziehend

\section{Diskussion}

Unsere Ergebnisse zeigen, dass die häusliche Leseumwelt einen positiven Effekt auf die kognitive und sprachliche Entwicklung sowohl türkischstämmiger als auch sehr früh und/oder sehr leicht geborener Kinder ausübt. Dies bedeutet, dass hinreichend gebildete Eltern von Vorschulkindern Resilienz fördern können, indem sie eine reichhaltige Leseumwelt zur Verfügung stellen.

Dies gilt jedoch nur bedingt für diejenigen Kinder, deren Mütter weniger als zehn Jahre eine Schule besucht haben, die also mit einem zusätzlichen sozioökonomischen Risiko aufwachsen. Unsere Vorgängerstudien (Jaekel, et al. 2011; Leyendecker et al. 2011) haben gezeigt, dass die Qualität der häuslichen Leseumgebung stark vom SES bzw. Bildungshintergrund der Eltern beeinflusst ist. Kinder, die einen türkischen Migrationshintergrund haben und deren Mütter wenig gebildet sind, erhalten zu Hause gege- 
benenfalls nicht die Lerngelegenheiten, die sie benötigen, um ihr volles Entwicklungspotential zu entfalten. Ähnliches gilt für Frühgeborene die in Familien mit niedrigem SES aufwachsen, da sie oftmals zusätzliche Förderung benötigen, um Entwicklungsrückstände aufzuholen, diese zu Hause aber nicht immer erhalten. Wie zuvor von Schneider et al. (2004) für früh und unreif geborene Kinder beschrieben, scheint der Einfluss eines niedrigen SES auf die kindliche Entwicklung sowohl in Gruppen mit biologischem wie auch mit soziokulturellem Risiko ähnlich zu sein.

Es ist zu vermuten, dass nicht nur die Quantität, sondern insbesondere auch die Qualität des familiären Leseverhaltens verantwortlich für den positiven Einfluss auf die kindliche Entwicklung ist (Leseman/de Jong 1998; Leseman/van den Boom 1999). Sollte sich dies in zukünftigen Studien bestätigen, so ist zu vermuten, dass das Leseverhalten in Familien mit niedriger Bildung eine andere Qualität (Art der Interaktionen beim Vorlesen, Auswahl des Lesematerials) hat, als in Familien mit mittlerem oder hohem Bildungsstand. Dies würde nicht nur die aktuellen Ergebnisse erklären, sondern auch wichtige Ansatzpunkte für Interventionen liefern, um Eltern mit niedrigem Bildungsstand eine bessere Förderung ihrer Kinder zu ermöglichen.

Darüber hinaus zeigten unsere Analysen unterschiedliche Effekte der Kontrollvariablen: obwohl der Einfluss des Geschlechts auf die kindliche Entwicklung nicht stabil signifikant war, deutete sich an, dass sehr früh und/oder sehr leicht geborene Jungen ein zusätzliches Risiko trugen, während türkischstämmige Mädchen tendenziell schlechter in den Tests abschnitten. Dies könnte ein Hinweis auf verschiedenartige Entwicklungsmechanismen abhängig von der Art des Risikos sein.

\section{Untersuchungsbegrenzung}

In der vorliegenden Studie verglichen wir Daten aus zwei Längsschnittstudien, um zu untersuchen, wie stark die familiäre Leseumwelt biologische und soziokulturelle Risiken kompensieren kann. Leider lassen unsere Ergebnisse jedoch nur sehr eingeschränkte Schlussfolgerungen zu, da Design und Stichprobencharakteristika trotz der versuchten Parallelisierung der Analysen erhebliche Unterschiede aufweisen. Zum Beispiel sah der ursprüngliche Analyseplan vor, gemeinsame Modelle für beide Bildungsgruppen sowie Interaktionseffekte (Bildung $\mathrm{x}$ häusliche Leseumwelt) zu berechnen. Leider wurde dieses Vorgehen der Datenstruktur der türkischstämmigen Stichprobe aufgrund der hohen Kollinearität zwischen mütterlichem Bildungshintergrund und HLE-Score nicht gerecht. Darüber hinaus sind die Zusammenhangsmuster in der türkischstämmigen Gruppe mit niedrigem Bildungshintergrund typischerweise sehr heterogen, da ein niedriger Bildungsstand hier weder mit der Bildungsmotivation noch mit der Intelligenz korreliert, sondern auf fehlende Bildungsopportunitäten im Heimatland zurückzuführen ist. Aus diesem Grund wurden die Regressionsanalysen separat durchgeführt, um die Effekte des HLE in den einzelnen Gruppen zeigen zu können. Einen anderen Faktor stellt der Einfluss der Stichprobengröße auf die Signifikanz der Regressionsanalysen dar: sowohl bei den türkischstämmigen als auch bei den sehr früh und/oder sehr leicht geborenen Kindern war die Teilnehmerzahl in den Subgruppen mit niedriger mütterlicher Bildung nur ungefähr ein Drittel so groß wie in den Subgruppen mit mittlerer mütterlicher Bildung. Wahrscheinlich 
wären die Effekte der häuslichen Leseumwelt für die sprachliche Entwicklung in den beiden Subgruppen mit niedriger mütterlicher Bildung bei entsprechender Stichprobengröße signifikant geworden.

Darüber hinaus möchten wir anmerken, dass wir in dieser Studie von Entwicklung sprechen, obwohl die Zusammenhänge zwischen HLE und kindlichem Entwicklungsstand bei den sehr früh und/oder sehr leicht geborenen Kindern nur querschnittlich untersucht wurden.

\section{Schlussfolgerungen}

Eine anregende familiäre Leseumwelt kann sowohl biologische als auch soziokulturelle Entwicklungsrisiken kompensieren. Eltern können ihre Kinder aktiv fördern, indem sie ihnen regelmäßig vorlesen. Für die Entwicklung erfolgreicher Interventionen müssen wir jedoch die spezifischen kindlichen Bedürfnisse kennen. Obwohl unsere Befunde vielversprechend sind, benötigen wir zusätzliche Studien zum Einfluss unterschiedlicher Risiken und protektiver Faktoren auf die kindliche Resilienz. Insbesondere Forschungsprojekte, die in ihrem Stichprobendesign das Vorhandensein verschiedener Entwicklungsrisiken berücksichtigen, kontrollieren und kombinieren, zum Beispiel indem Frühgeborene mit Migrationshintergrund langfristig untersucht werden, wären äußerst wünschenswert.

\section{Literatur}

Aarnoudse-Moens, C., Weisglas-Kuperus, N., van Goudoever, J. \& Oosterlaan, J. (2009). Meta-analysis of neurobehavioral outcomes in very preterm and/or very low birthweight children. Pediatrics, 124, 2, S. 717-728.

Arnold, D. H., \& Doctoroff, G. L. (2003). The early education of socioeconomically disadvantaged children. Annual Review of Psychology, 54, S. 517-545.

Belsky, J. (1997). Theory testing, effect size evaluation, and differential susceptibility to rearing influence: the case of mothering and attachment. Child Development, 64, 4, S. 598-600.

Belsky, J. \& Pluess, M. (2009). Beyond diathesis-stress: Differential susceptibility to environmental influences. Psychological Bulletin, 135, 6, S. 885-908.

Bradley, R. H., Whiteside, L., Mundfrom, D. J., Blevins-Knabe, B., Casey, P. H., Caldwell, B. M., Kelleher, K. H., Pope, S. \& Barrett, K. (1995). Home environment and adaptive social behavior among premature, low birth weight children: Alternative models of environmental action. Journal of Pediatric Psychology, 20, 3, S. S. 347-362.

Bus, A. G., Leseman, P. P. M. \& Keultjes, P. (2000). Joint book reading across cultures: A comparison of Surinamese-Dutch, Turkish-Dutch, and Dutch parent-child dyads. Journal of Literacy Research, 32,1, S. 53-76.

Bus, A. G. \& Sulzby, E. (1996). Becoming literate in a multicultural society. In: Shimron, J. (Hrsg.), Literacy and education: Essays in memory of Dina Feitelson. Creskill, NJ: Hampton Press, S. 17-32.

Bus, A. G., van Ijzendoorn, M. H. \& Pellegrini, A. D. (1995). Joint book reading makes for success in learning to read: A meta-analysis on intergenerational transmission of literacy. Review of Educational Research, 65, S. 1-21.

Caspar, U. \& Leyendecker, B. (2011). Deutsch als Zweitsprache. Zeitschrift für Entwicklungspsychologie und Pädagogische Psychologie, 43, 3, 118-132. DOI: 10.1026/0049-8637/a000046.

Cicchetti, D. (2010). Resilience under conditions of extreme stress: A multilevel perspective. World Psychiatry: Official Journal of the World Psychiatric Association (WPA), 9, 3, S. 145-154. 
Citlak, B., Leyendecker, B., Harwood, R. L. \& Schölmerich, A. (2008). Long-term socialization goals of first and second generation migrant Turkish mothers and German mothers. International Journal of Behavioral Development, 32, S. 57-66.

DeWolff, M. S. \& van Ijzendoorn, M. H. (1997). Sensitivity and attachment: A meta-analysis on parental antecedents of infant attachment. Child Development, 68, 4, S. 571-591.

Driessen, R., Leyendecker, B., Schölmerich, A. \& Harwood, R. (2009). Everyday experiences of 18- to 36-month-old children from migrant families: The influence of host culture and migration experience. Early Child Development and Care, First DOI: 10.1080/03004430902872182.

Feldman, R. (2007). Maternal versus child risk and the development of parent-child and family relationships in five high-risk populations. Development and Psychopathology, 19, S. 293-312.

Forcada-Guex, M., Pierrehumbert, B., Borghini, A., Moessinger, A. \& Muller-Nix, C. (2006). Early dyadic patterns of mother-infant interactions and outcomes of prematurity at 18 months. Pediatrics, 118,1 , S. e107-e114.

Glick, J. E., Bates, L. \& Yabiku, S. T. (2009). Mother's age at arrival in the United States and early cognitive development. Early Childhood Research Quarterly, 24, 4, S. 367-380. DOI: 10.1016/ j.ecresq.2009.01.001:

Gogolin, I., Neumann, U. \& Roth, H.-J. (2003). Förderung von Kindern und Jugendlichen mit Migrationshintergrund. Bonn: Bund-Länder-Kommission zur Bildungsplanung und Forschungsförderung (Materialien zur Bildungsplanung und zur Forschungsförderung. Heft 107).

Grimm, H., Aktas, M. \& Frevert, S. (2001). SETK 3-5. Sprachentwicklungstest für drei- bis fünfjährige Kinder. Diagnose von Sprachverarbeitungsfähigkeiten und auditiven Gedächtnisleistungen. Göttingen: Hogrefe.

Grimm, H. \& Schöler, H. (1991). Der Heidelberger Sprachentwicklungstest H-S-E-T. Göttingen: Hogrefe (2. korrigierte Auflage).

Hall, J., Jaekel, J. \& Wolke, D. (2012, in Druck). Gender distinctive impacts of prematurity and small for gestational age on age 6 attention problems. Child and Adolescent Mental Health.

Jaekel, J., Schölmerich, A., Kassis, W. \& Leyendecker, B. (2011). Parental bookreading as a resource for pre-schoolers' cognitive skills in Turkish migrant and German non-migrant families. International Journal of Developmental Science, 5, S. 27-39.

Jaekel, J., Wolke, D. \& Bartmann, P. (2012, in Druck). Poor attention rather than hyperactivity/ impulsivity predicts academic achievement in very preterm and fullterm adolescents. Psychological Medicine.

Johnson, S. (2007). Cognitive and behavioural outcomes following very preterm birth. Seminars in Fetal and Neonatal Medicine, 12, 5, S. 363-373.

Johnson, S., Hennessy, E., Smith, R., Trikic, R., Wolke, D. \& Marlow, N. (2009). Academic attainment and special educational needs in extremely preterm children at 11 years of age: The EPICure study. Archives of Disease in Childhood - Fetal and Neonatal Edition, 94, 4, S. F283-F289. DOI: 10.1136/ adc.2008.152793.

Jordan, N. C. \& Levine, S. C. (2009). Socioeconomic variation, number competence, and mathematics learning difficulties in young children. Developmental Disabilities Research Reviews, 15, 1, 60-68. DOI: $10.1002 /$ ddrr.46.

Kaufman, A. \& Kaufman, N. (1983). Kaufman Assessment Battery for Children. Circle Pines, MN: American Guidance Service.

Kim, Y. S. (2009). The relationship between home literacy practices and developmental trajectories of emergent literacy and conventional literacy skills for Korean children. Reading and Writing, 22; 1 , S. 57-84. DOI: $10.1007 / \mathrm{s} 11145-007-9103-9$.

Laucht, M., Esser, G. \& Schmidt, M. H. (2002). Vulnerability and resilience in the development of children at risk: the role of early mother-child interaction. Revista de Psiquiatria Clínica, 29, 1, S. 20-27.

Leseman, P. P. M. \& de Jong, P. F. (1998). Home literacy: Opportunity, instruction, cooperation and social-emotional quality predicting early reading achievement. Reading Research Quarterly, 33, 3, S. 294-318. 
Leseman, P. P. M. \& van den Boom, D. C. (1999). Effects of quantity and quality of home proximal processes on Dutch, Surinamese-Dutch and Turkish-Dutch pre-schoolers' cognitive development. Infant and Child Development, 8, 1, S. 19-38. DOI: 10.1002/(sici)1522-7219(199903)8:1<19::aidicd187>3.0.co;2-7.

Leyendecker, B. (2003). Die frühe Kindheit in Migrantenfamilien. In: Keller, H. (Hrsg.), Handbuch der Kleinkindforschung. Bern: Verlag Hans Huber, S. 381-431 (3. Auflage).

Leyendecker, B., Jaekel, J., Olcay Kademoglu, S. \& Yagmurlu, B. (2011). Parenting practices and preschoolers' cognitive skills in Turkish immigrant and German families. Early Child Development and Care, 181, 8, S. 1095-1110.

Leyendecker, B. \& Schölmerich, A. (2005). Familie und kindliche Entwicklung im Vorschulalter: Der Einfluss von Kultur und sozioökonomischen Faktoren. In Fuhrer, U. \& Uslucan, H.-H. (Hrsg.), Familie, Akkulturation und Erziehung. Migration zwischen Eigen- und Fremdkultur. Stuttgart: Verlag W. Kohlhammer, S. 17-39.

Leyendecker, B., Schölmerich, A. \& Citlak, B. (2006). Similarities and differences between first- and second-generation Turkish migrant mothers in Germany: The acculturation gap. In Bornstein, M. H. \& Cote. L. R. (Hrsg.), Acculturation and parent-child relationships: Measurement and development. Mahwah, NJ: Erlbaum, S. 297-315.

Masten, A. S. (2007). Resilience in developing systems: Progress and promise as the fourth wave rises. Development and Psychopathology, 19, 3, S. 921-930. DOI: 10.1017/s0954579407000442

Melchers, P. \& Preuss, U. (1991). K-ABC: Kaufman Battery for Children: Deutschsprachige Fassung. Frankfurt, AM: Swets \& Zeitlinger.

Mol, S. E., Bus, A. G., De Jong, M. T. \& Smeets, D. J. H. (2008). Added value of dialogic parent-child book readings: A meta-analysis. Early Education \& Development, 19, 1, S. 7-26.

Mulder, H., Pitchford, N. J. \& Marlow, N. (2010). Processing speed and working memory underlie academic attainment in very preterm children. Archives of Disease in Childhood - Fetal and Neonatal Edition, 95, 4, S. F267-F272. DOI: 10.1136/adc.2009.167965.

Nosarti, C., Murray, R. M. \& Hack, M. E. (2010). Neurodevelopmental outcomes of preterm birth. Cambridge: Cambridge University Press.

Petermann, F., Stein, I. A. \& Macha, T. (2006). ET 6-6. Entwicklungstest 6 Monate bis 6 Jahre. Frankfurt: Pearson (3., veränderte Auflage).

Quigley, M., Poulsen, G., Boyle, M., Wolke, D., Field, D., Alfirevic, Z. \& Kurinczuk, J. J. (2012). Early term and late preterm birth is associated with poorer school performance at age 5 years: A cohort study. Archives of Disease in Childhood - Fetal and Neonatal Edition. DOI: 10.1136/archdischild2011-300888.

Raikes, H., Pan, B. A., Luze, G., Tamis-LeMonda, C. S., Brooks-Gunn, J., Constantine, J., Banks Tarullo, L., Raikes, H. A. \& Rodriguez, E. T. (2006). Mother-child bookreading in low-income families: correlates and outcomes during the first three years of life. Child Development, 77, 4, S. 924-953.

Rees, S. \& Inder, I. (2005). Fetal and neonatal origins of altered brain development. Early Human Development, 81, S. 753-761.

Riegel, K., Ohrt, B., Wolke, D. \& Österlund, K. (1995). Die Entwicklung gefährdet geborener Kinder bis zum fünften Lebensjahr. Die Arvo Ylppö-Neugeborenen-Nachfolgestudie in Südbayern und Südfinnland. Stuttgart: Ferdinand Enke Verlag.

Saigal, S., van Ouden, L., Wolke, D., Hoult, L., Paneth, N., Streiner, D. L., Whitaker, A. \& Pinto-Martin, J. (2003). School-age outcomes in children who were extremely low birth weight from four international population-based cohorts. Pediatrics, 112, 4, S. 943-950.

Schneider, W., Wolke, D., Schlagmüller, M. \& Meyer, R. (2004). Pathways to school achievement in very preterm and full term children. European Journal of Psychology of Education, 19, 4, S. 385-406.

Schölmerich, A., Leyendecker, B., Citlak, B., Caspar, U. \& Jäkel, J. (2008). Testing minority and migrant children. Journal of Psychology, 216, S. 187-194.

Taylor, H. G., Klein, N., Anselmo, M. G., Minich, N., Espy, K. A. \& Hack, M. (2011). Learning problems in kindergarten students with extremely preterm birth. Archives of Pediatrics \& Adolescent Medicine, 165, 9, S. 819-825. DOI: 10.1001/archpediatrics.2011.137 
Weisglas-Kuperus, N., Baerts, W., Smrkovsky, M. \& Sauer, P. J. J. (1993). Effects of biological and social factors on the cognitive development of very low birth weight children. Pediatrics, 92, 5, S. 658-665.

Whitehurst, G. J., Arnold, D. S., Epstein, J. N., Angell, A. L., Smith, M. \& Fischel, J. (1994). A picture book reading intervention in day care and home for children from low-income families. Developmental Psychology, 30, S. 679-689.

Wolke, D. (1998). Psychological development of prematurely born children. Archives of Disease in Childhood, 78, S. 567-570.

Wolke, D. \& Meyer, R. (1999). Cognitive status, language attainment, and prereading skills of 6-yearold very preterm children and their peers: The Bavarian Longitudinal Study. Developmental Medicine \& Child Neurology, 41, S. 94-109.

Wolke, D., Schmid, G. M., Schreier, A. P. \& Meyer, R. D.-P. (2009). Crying and feeding üroblems in infancy and cognitive outcome in preschool children born at risk: A prospective population study. Journal of Developmental \& Behavioral Pediatrics, 30,3 , S. 226-238.

Wolke, D., Schulz, J. \& Meyer, R. (2001). Entwicklungslangzeitfolgen bei ehemaligen, sehr unreifen Fruehgeborenen. Monatsschrift Kinderheilkunde, 149, Suppl.1, S. 53-61.

Eingereicht am/Submitted on: 14.03.2012

Angenommen am/Accepted on 06.07.2012

Anschrift der Autorinnen und des Autors/Addresses of the authors:

Dr. Julia Jäkel (Korrespondenzautorin/corresponding author)

Ruhr-Universität Bochum

Fakultät Psychologie

AE Entwicklungspsychologie

44780 Bochum

Deutschland/Germany

Prof. Dr. Dieter Wolke

The University of Warwick

Department of Psychology and Division of Mental Health and Wellbeing

Warwick Medical School

Coventry CV4 7AL

Vereinigtes Königreich/United Kingdom

Prof. Dr. Birgit Leyendecker

Ruhr-Universität Bochum

Fakultät Psychologie

AE Entwicklungspsychologie

44780 Bochum

Deutschland/Germany

E-Mail: julia.jaekel@rub.de

D.Wolke@warwick.ac.uk

birgit.leyendecker@rub.de 